\title{
Control of Phase-Separated Structure in (Polymer/Liquid Crystal) Composite Films and Their Electro-Optical Switching Characteristics
}

\author{
Hirotsugu KikUChI, Fumihiko Usui, and Tisato KaJIYama* \\ Department of Chemical Science and Technology, Faculty of Engineering, Kyushu University, \\ 6-10-1 Hakozaki, Higashi-ku, Fukuoka 812, Japan
}

(Received August 28, 1995)

\begin{abstract}
Phase-separation behavior and electro-optical properties were investigated for the composite films consisting of the three dimensional polymer network and low molecular weight liquid crystals (LCs) embedded in the network. The composite films were prepared by a solvent cast method from uniform solutions. It was confirmed from solvent-evaporation time dependence of light scattering that the phase-separated structure of the polymer and LC components was formed via the spinodal decomposition. The resultant domain size of LC in the composite film was controlled by regulating the solvent evaporation rate. The electro-optical properties based on the electric field controlled-light scattering of the composite film was strongly dependent on the LC domain size. With increasing the LC domain size in the composite film, the rise and decay response speeds were increased and decreased, respectively, due to a decrease in an interfacial interaction between the LC and polymer phases.

KEY WORDS (Polymer/Liquid Crystal) Composite Film / Phase-Separation / Spinodal Decomposition / Light Scattering / Electro-Optical Switching /
\end{abstract}

A co-continuous composite film consisting of the polymer and the low molecular weight liquid crystal (LC) was developed to apply unique characteristics of LC as a self-supported flexible film. ${ }^{1-4}$ The LC is embbeded in the three dimensional polymer networks in the composite film prepared by a solvent cast method. ${ }^{3-7}$ Since the (polymer/LC) composite film exhibits reversible light scattering-light transmission switching under conditions of off- and on- a.c. electric fields, it is expected to be applied to the large area and flexible display or light control valve..$^{8-19}$ The light scattering state is dependent on optical heterogeneties in the composite film, such as a spatial distortion of nematic directors and/or mismatching in refractive indices of the components. ${ }^{15}$ Consequently, the light scattering and light transmission switching properties of the composite film are decisively influenced by the phase separated structure in the composite film. It is, therefore, necessary to study the phase separation mechanism during the solvent evaporation to control the phase-separated structure of the composite film.

In this paper, a mechanism of polymer and LC phase-separation, a control of the LC domain size in the (polymer/LC) composite film, relationships between the LC domain size and electro-optical properties such as response speeds, the light transmittance and the driving voltage have been investigated.

\section{EXPERIMENTAL}

\section{Materials}

The chemical structures of the constituent polymers and LC materials for the composite films are given in Figure 1. Poly(diisopropyl fumarate) (Pdi-iPF) was used as the matrix polymers. E8 (purchased from BDH Chemical Co., Ltd.) was used as the LC materials. E8 is a nematic mixture consisting of several cyanophenyl type LCs.

\section{Preparation of the Composite Film and Light Scattering Measurement}

The composite films were cast from a chloroform solution of a mixture of polymer and LC materials under the conditions of the constant temperature and the constant evaporation rate using a home-made equipment as shown in Figure 2. This setup involves the measuring systems of the light scattering intensity and the weight change in the solution during the film formation process. The weight change was monitored using a semiconductor strain gauge. The light scattering and light transmittance properties were studied using $\mathrm{He}-\mathrm{Ne}$ gas laser (wavelength of $632.8 \mathrm{~nm}$, Uniphase) as an incident light beam. The transmitted light intensity was measured with a photodiode which was positioned at the direction of incident beam. The scattered intensity profile from the cast solution was detected with a photodiode array. The solvent evaporation rate was controlled by regulating the pressure in the solvent evaporation chamber with a needle valve and a vacuum pump. The weight ratio of polymer/LC was $40 / 60$ and the initial

1) Polymer

$$
\begin{aligned}
& \text { Poly (diisopropyl fumarate) } \quad \mathrm{CH}_{3}
\end{aligned}
$$

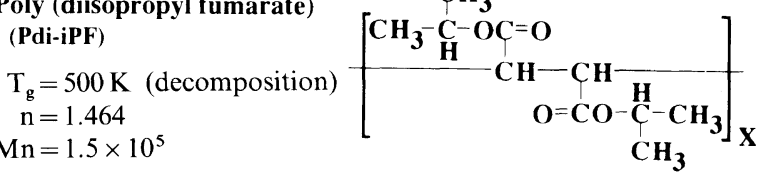

2) Liquid crystal

E8 $(\mathrm{BDH})$ nematic mixture consisting of cyanophenyl LCs

$$
\begin{aligned}
\mathrm{T}_{\mathrm{NI}} & =345 \mathrm{~K} \\
\mathrm{n} \perp & =1.527 \mathrm{n}_{/ /}=1.774 \\
\Delta \mathrm{n} & =0.247 \mathrm{n}_{\mathrm{AVE}}=1.609
\end{aligned}
$$

Figure 1. The chemical structures of the constituent polymers and LC materials for the composite films

\footnotetext{
* To whom correspondence should be addressed.
} 


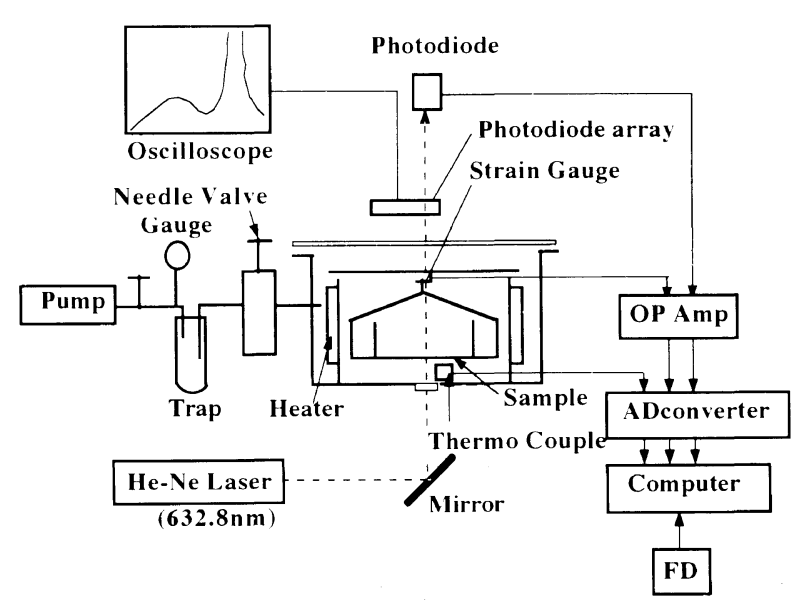

Figure 2. Measuring system of light scattering intensity profile and weight change in the (polymer/LC) solution during the film formation process.

concentration of the solution was $3-8 \mathrm{wt} \%$. The thickness of the composite films finally obtained ranged $10-20 \mu \mathrm{m}$.

\section{Scanning Electron Microscopic (SEM) Observation}

The aggregation state of the composite film was investigated by means of scanning electron microscope (SEM, Hitachi S-430). The composite film was fractured in liquid nitrogen and then, LC phase in the (Pdi-iPF/LC) composite film was extracted with $n$-hexane at room temperature. The film and fracture surfaces were coated with gold for the SEM observation.

\section{Electro-Optical Measurement}

In order to evaluate the electro-optical properties of the composite film, light transmittance changes upon the application of an a.c. electric field were studied by an experimental setup as shown in Figure 3. The composite films were sandwiched between two indium/tin oxide (ITO) coated glasses. He-Ne laser was used as an incident light source. The measurement of the transmitted light intensity through the composite films without any polarizers was carried out with a photodiode under the modulation of an electric field. The transmitted light intensity through the sample cell was normalized by the intensity through a blank cell. The transient changes in the transmitted light intensity upon the application and the removal of an electric field were also recorded with a digital storage oscilloscope (Kawasaki KDS-102).

\section{RESULTS AND DISCUSSION}

\section{Formation Process of Phase-Separated Structure in} (Polymer/LC) Composite Film

The phase-separated structure of the (polymer/LC) composite film is formed during the solvent evaporation from a uniform solution. In order to investigate how the phase-separation occurs, the light scattering properties during solvent evaporation was measured. Figure 4 shows the time dependence of the transmitted light intensity and the weight change in the chloroform solution of Pdi-iPF and E8 at $308 \mathrm{~K}$. Though the weight of the solution decreased monotonously, the transmittance uncontinuously decreased in steps. Then, the solvent

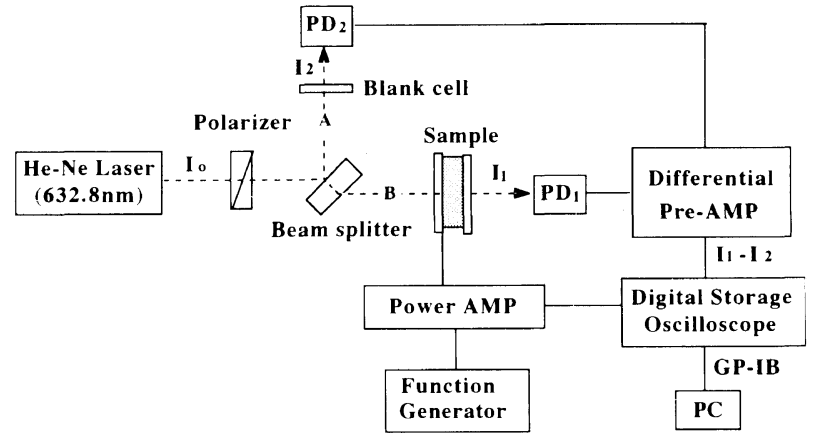

Figure 3. Schematic diagram of the measuring system of electrooptical properties of the composite film.

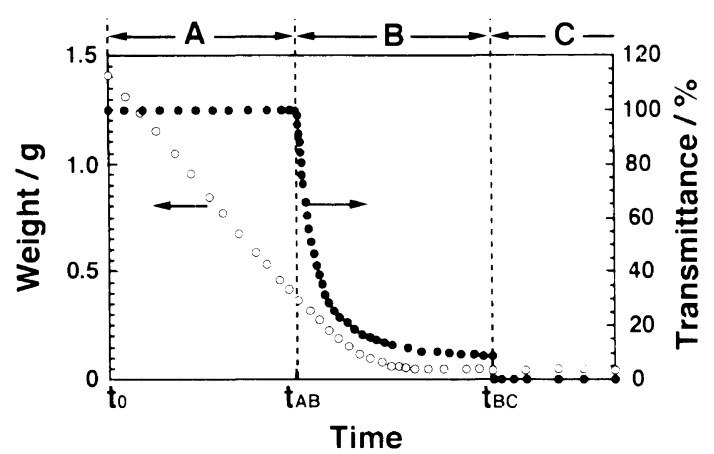

Figure 4. The time dependence of the transmitted light intensity and the weight change in the chloroform solution of Pdi-iPF and E8 at $308 \mathrm{~K}$.

evaporation process can be divided into three stages $\mathrm{A}$, $\mathrm{B}$, and $\mathrm{C}$, based on the transmittance changes. In the stage $\mathrm{A}$, the solution was almost clear and no change of the transmittance was observed. The transmittance decreased abruptly at the beginning of the stage B and was gradually approaching about $10 \%$. At the beginning of the stage $\mathrm{C}$, the transmittance decreased up to nearly $0 \%$ uncontinuously. Here, $t_{0}, t_{\mathrm{AB}}$, and $t_{\mathrm{BC}}$ denote the start times of each stage of $\mathrm{A}, \mathrm{B}$, and $\mathrm{C}$, respectively. $t_{\mathrm{AB}}-t_{0}$ and $t_{\mathrm{BC}}-t_{0}$ were about $1100 \mathrm{~s}$ and $2200 \mathrm{~s}$, respectively, in the case of the initial solution weight of $1.4 \mathrm{~g}$ and the evaporation rate of $3.2 \mathrm{~g} \mathrm{~h}^{-1}$ at $308 \mathrm{~K}$. The evaporation rate was determined from the slope of the weight $v s$. time curve at $t_{\mathrm{AB}}$. The reduction of the transmittance with time corresponds to the generation of light scattering in the solution, that is, the appearance of phase-separated structure with increasing the concentration of the solution. In the stage A, the solution was optically homogeneous and did not have any light scattering factor without a thermal fluctuation of the solution density. Since no optical anisotropy was observed and also there remained enough amount of solvent at the beginning of the stage $\mathrm{B}$, it is clear that the optically isotropic phases were separated in the solution during the stage B. An optical anisotropy was recognized in the stage $\mathrm{C}$ based on the polarized optical analysis under crossed nicols. This result makes it clear that the LC phase of E8 appeared in the stage C. Therefore, it is reasonable to conclude from the transmittance behavior in the stages $\mathrm{B}$ and $\mathrm{C}$ that the appearance of the anisotropic E8 phase does not play an important role in the generation of the phase-separation. Though the chloroform is a goodsolvent for both Pdi-iPF and E8, Pdi-iPF and E8 are not 


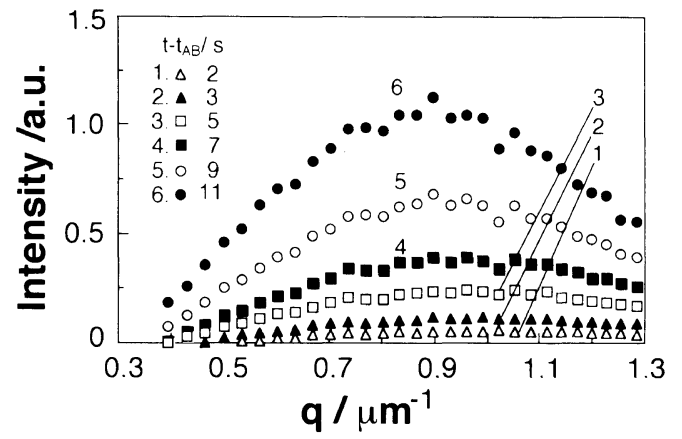

(a)

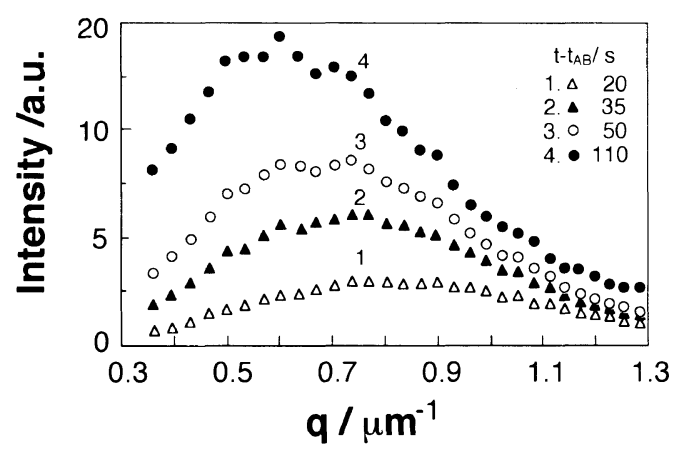

(b)

Figure 5. Variation of scattering vector, $q$ dependence of light scattering intensity with time of solvent evaporation for the (Pdi-iPF/E8: 40/60) solution in the stage B.

compatible each other. With increasing the concentration of the solution, the Pdi-iPF-rich phase and the E8-rich one are separated in the solution, because they prefer to be excluded each other. In other words, the E8-rich phase must serve as a poor-solvent to Pdi-iPF. In the end of the stage $\mathrm{B}$, these phases might mainly consist of the solid-like Pdi-iPF and the isotropic E8 phases since most of the solvent evaporated. After furthermore evaporation of solvent, the isotropic phase of E8 transforms into the nematic phase, resulting in the generation of remarkably strong light scattering due to the random orientation of the nematic directors in the stage C.

Light scattering behavior with the maximum scattering angle was recognized in the stage $\mathrm{B}$. This apparently indicates that a periodic structure of the Pdi-iPF-rich phase and the E8-rich one was generated in the solution in the stage $\mathrm{B}$. The strong and broad scattering due to the multiple scattering of the anisotropic LC phases was observed in the stage $\mathrm{C}$. The light scattering profiles in the stage $\mathrm{B}$ are shown in Figure 5 as a function of the scattering vector $q=(4 \pi / \lambda) \sin (\theta / 2)$, where $\lambda$ and $\theta$ are the wavelength of incident light and the scattering angle, respectively. An increase in the maximum intensity of scattering with existing time in the stage $\mathrm{B}, t-t_{\mathrm{AB}}$ would reflect an increase in the difference between refractive indices of the Pdi-iPF-rich phase and the E8-rich one. The plot of the maximum scattering vector, $q_{\mathrm{m}}$ vs. $t-t_{\mathrm{AB}}$ is shown in Figure 6 . For initial $10 \mathrm{~s}$ in the stage $\mathrm{B}$, the magnitude of $q_{\mathrm{m}}$ showed almost constant though the scattering intensity increased in this period. After $10 \mathrm{~s}$ of $t-t_{\mathrm{AB}}$, the $q_{\mathrm{m}}$ started to decrease monotonously. The magnitude of $q_{\mathrm{m}}$ corresponds to the

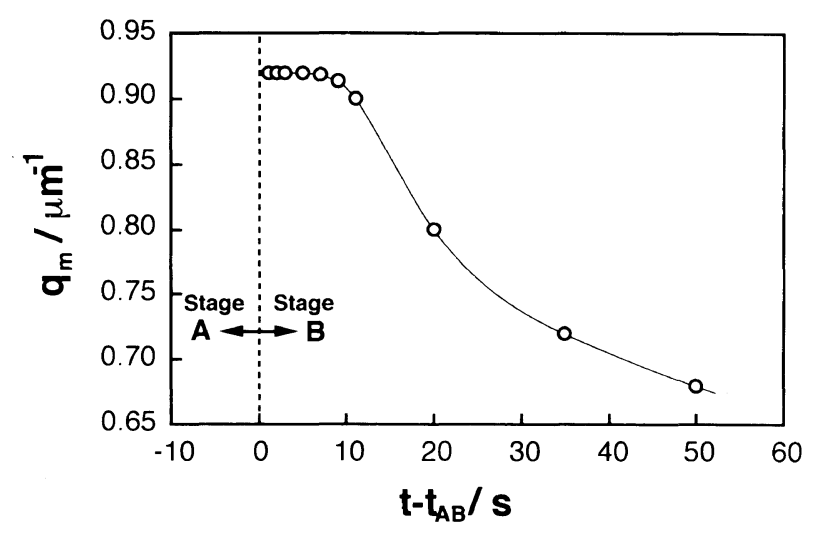

Figure 6. The time dependence of the maximum scattering vector, $q_{\mathrm{m}}$ from $t_{\mathrm{AB}}$ for the (Pdi-iPF/E8: $\left.40 / 60\right)$ solution in the stage B.

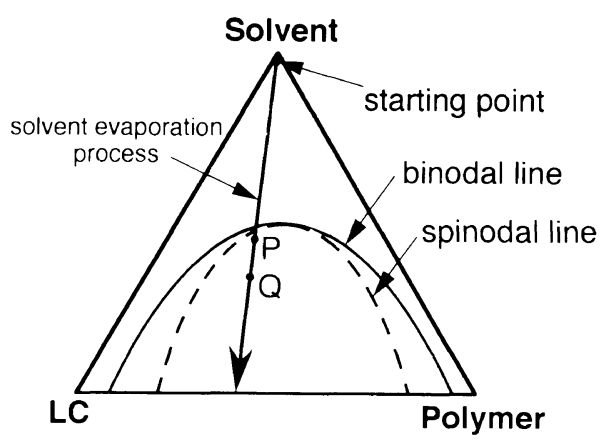

Figure 7. Hypothetical phase diagram of the (polymer/ LC/solvent) ternary system. The arrow in the diagram shows a route of the solvent evaporation process.

reciprocal of the periodicity of phase-separated structure. Therefore, the result in Figure 6 means that the periodicity of phase-separated structure composed of the Pdi-iPF-rich phase and the E8-rich one was constant at the early period in the stage $B$ and then increased. There are two major mechanisms by which two components of a mutual solution can phase-separate; (1) nucleationgrowth, and (2) spinodal decomposition. The nucleationgrowth mechanism results in phase-separated domain size increasing with time. On the other hand, in the case of the spinodal decomposition mechanism, the domains are of about the same size during the early stages of phase separation. ${ }^{20,21}$ The light scattering behavior observed at the early period in the stage B agrees to the kinetics of the spinodal decomposition. In the stage $\mathrm{C}$, though the intensity of light scattering remarkably increased, the scattering maximum disappeared because of the strong multiple scattering from the nematic phase. It can be apparently concluded from the light scattering behavior mentioned above that the phase separation in the Pdi-iPF/E8 solution starts at $t_{\mathrm{AB}}$ through the spinodal decomposition, and then the domains may coalesce each other, forming larger phase-separated structure with time.

The phase separation behavior of the (polymer/LC) composite system in a solvent evaporation process can be explained on the basis of the phase diagram as schematically shown in Figure 7, which is a hypothetical phase diagram of the (Pdi-iPF/E8/chloroform) ternary system. The solid and broken curves in the phase diagram correspond to the binodal and spinodal lines, respectively. The binodal line separates the phase region 

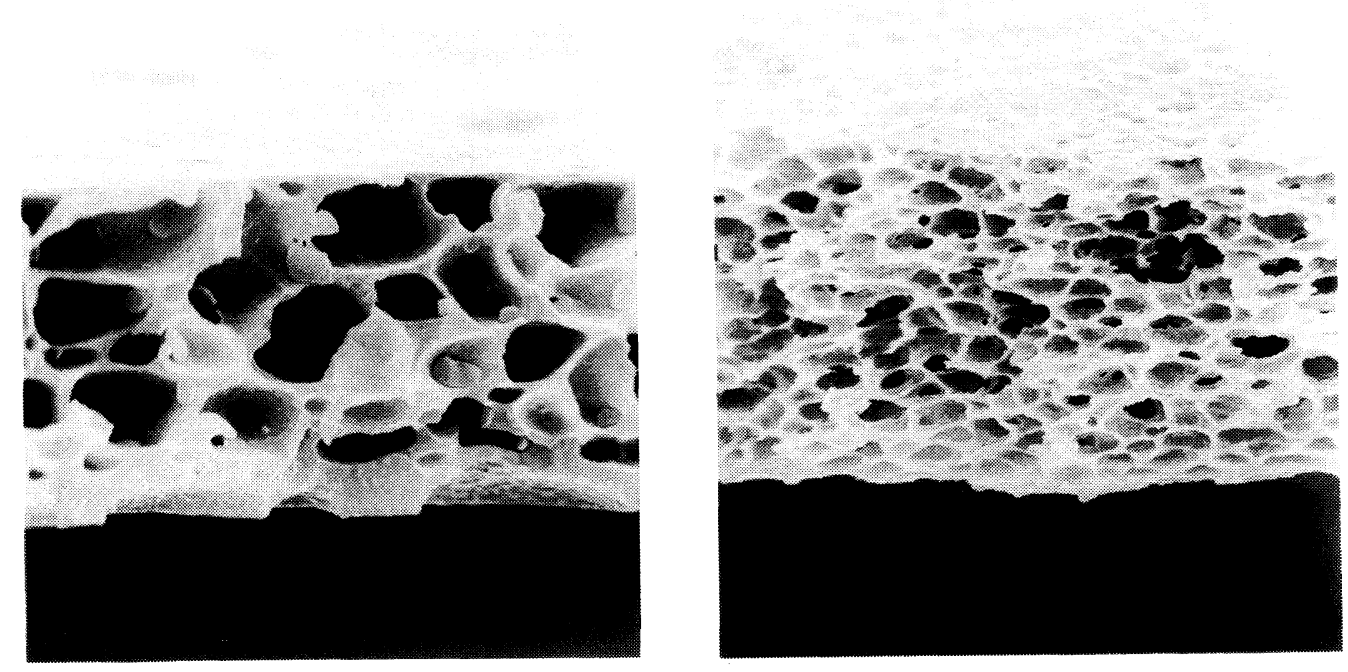

(a)

\section{$10 \mu \mathrm{m}$}

(b)

Figure 8. SEM photographs of the matrix Pdi-iPFs which were prepared at the solvent evaporation rates of (a) $1.8 \mathrm{gh}^{-1}$ and (b) $7.0 \mathrm{gh}^{-1}$, respectively.

into the homogeneous and the phase-separated regions. Also, the spinodal line separates the thermodynamically metastable and the unstable regions. In the metastable region which exists between the spinodal and binodal lines, an initially homogeneous solution is phaseseparated by the nucleation-growth mechanism. In the unstable region within the spinodal line, the phase separation proceeds via spinodal decomposition. During the solvent evaporation, the concentration of the solution changes along the straight line shown in Figure 7 . The solution should pass the metastable region before it thrusts into the unstable region. Since the nucleation and growth process requires an activation energy, the phase separation rate must be slow. On the other hand, the phase separation through the spinodal decomposition can proceed very fast because the energy barrier is negligible in the spinodal decomposition. ${ }^{20,21}$ Therefore, the solution should phase-separate preferentially via the spinodal decomposition. When the evaporation rate is fairly high, the phase-separation scarcely occur until the solution reaches a higher concentration region such as the point $\mathrm{Q}$. At the point $\mathrm{Q}$, being the further position from the spinodal line compared with the point $\mathrm{P}$, the decomposition rate is much faster and the periodicity of the modulated structure should be smaller than those at the point $\mathrm{P}$ according to Cahn's theory. ${ }^{20,21}$ Based on the spinodal decomposition mechanism, the resulting domain size should be larger with reducing the solvent evaporating rate. This prediction was confirmed by morphological studies of phase-separated structure for the composite films. Figures 8(a) and (b) show the SEM photographs of the matrix Pdi-iPFs which were prepared at the solvent evaporation rates of $1.8 \mathrm{~g} \mathrm{~h}^{-1}$ and $7.0 \mathrm{~g} \mathrm{~h}^{-1}$, respectively, and from which $\mathrm{LC}$ was extracted with n-hexane. It is apparent from Figure 8 that the larger E8 domains were formed in the case of the slower solvent

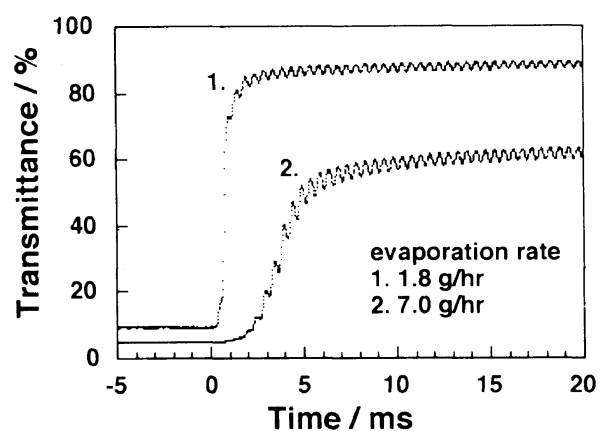

(a)

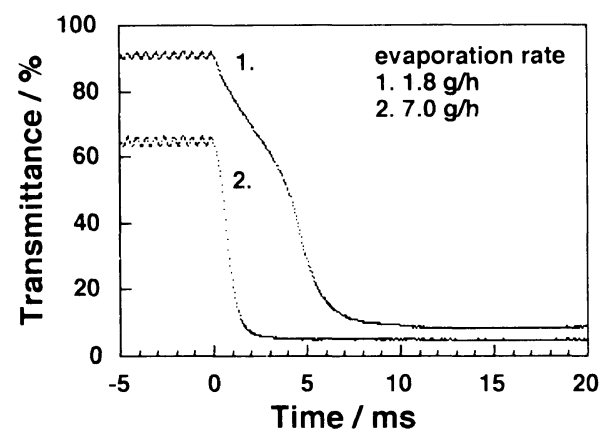

(b)

Figure 9. The rise and the decay response curves of the electro-optical switching for the (Pdi-iPF/E8: 40/60 wt\%) composite films prepared at evaporation rates of $1.8 \mathrm{~g} \mathrm{~h}^{-1}$ (curve 1) and $7.0 \mathrm{~g} \mathrm{~h}^{-1}$ (curve 2).

evaporation rate.

\section{Electro-Optical Switching of (Polymer/LC) Composite Film}

Figure 9 shows the rise and the decay response curves of transmittance for the (Pdi-iPF/E8:40/60 wt\%) compo- 
site films prepared at the different evaporation rates of $1.8 \mathrm{~g} \mathrm{~h}^{-1}$ and $7.0 \mathrm{~g} \mathrm{~h}^{-1}$. An applied a.c. electric voltage was $70 \mathrm{~V}_{\mathrm{rms}}$. The rise response for the composite film prepared at the evaporation rate of $1.8 \mathrm{~g} \mathrm{~h}^{-1}$ was faster than that of $7.0 \mathrm{~g} \mathrm{~h}^{-1}$. On the other hand, the decay response behavior were contrary to those of the rise one. When an electric field is applied to the liquid crystal surrounded by polymer walls or networks, three physical contributions, (1) the elastic modulus of nematic directors, (2) the anchoring effect at the (polymer/LC) interface, and (3) the dielectric torque due to the dielectric anisotropy of LC, should be exerted statically on the molecular alignment of LC. In the case of dynamic process, (4) the viscosity effect for the LC molecular reorientation must be furthermore taken into consideration. Since the LC used in this study possesses a positive dielectric anisotropy, the LC molecules tend to align along the direction of an applied electric field. The viscosity opposes the molecular reorientation in both cases of the rise and the decay processes. Though the anchoring effect resists the molecular reorientation along the direction of an applied electric field in the rise process, it enhances the random orientation of the LC directors in the decay process. Namely, the anchoring effect is contradictory exerted on the LC reorientation in the processes of the rise and decay, that is, acting as a resistance and a restoring force, respectively. The anchoring strength is proportional to the area of the (polymer/LC) interface. The area of the interface in the composite film prepared at the evaporation rate of $7.0 \mathrm{~g} \mathrm{~h}^{-1}$ should be much larger than that at $1.8 \mathrm{~g} \mathrm{~h}^{-1}$ because the size of LC domains shown in Figure 8(b) is apparently smaller than that in Figure 8(a). Therefore, it is reasonable to consider that the anchoring effect on LC molecular reorientation in the composite film prepared at the evaporation rate of $7.0 \mathrm{~g} \mathrm{~h}^{-1}$ is much larger than that at $1.8 \mathrm{gh}^{-1}$. The difference in the response speeds for the composite films prepared at $1.8 \mathrm{~g} \mathrm{~h}^{-1}$ and $7.0 \mathrm{~g} \mathrm{~h}^{-1}$ corresponds to the difference in the net strength of anchoring effects at the (polymer/LC) interface in the composite films. A shoulder can be recognized on the decay switching curve 1 shown in the Figure 9(b). This might be due to the presence of two steps of decay process for the composite film prepared at the evaporation rate of $1.8 \mathrm{~g} \mathrm{~h}^{-1}$ in which the size of LC domains was about 3-4 $\mu \mathrm{m}$. The field coherent length, ${ }^{22}$ which roughly corresponds to the thickness of anchored LC layer at the polymer wall depending on the magnitude of an electric field, might be the order of sub- $\mu \mathrm{m}$. Since the size of LC domains in the composite film prepared at $1.8 \mathrm{~g} \mathrm{~h}^{-1}$ is much larger than the coherent length as shown in Figure 8(a), it is reasonable to consider that the LC molecules in the vicinity of the polymer wall reorient in a shorter decay response process by the anchoring effect, and then the LC molecules existing in the center region of each domain reorient in a longer decay response process. On the other hand, no shoulder was detected in the decay switching curve 2 in Figure $9(\mathrm{~b})$. This might be due to that the LC domain size in the composite film prepared at the evaporation rate of $7.0 \mathrm{gh}^{-1}$ was enough small. Then, LC molecules could reorient at almost same time.

The large difference in the transmittance in the presence

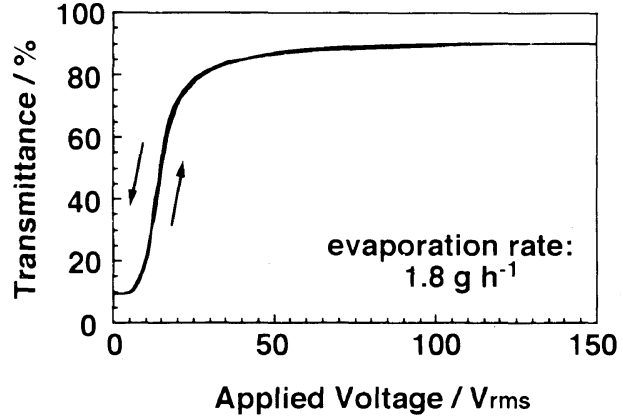

(a)

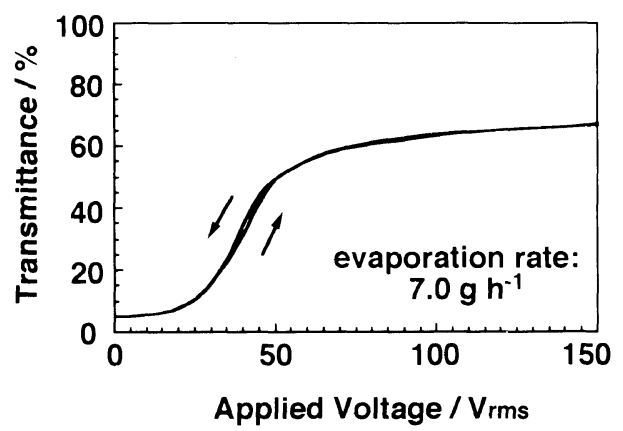

(b)

Figure 10. The applied voltage dependence of the transmittance for the (Pdi-iPF/E8: $40 / 60 \mathrm{wt} \%$ ) composite films prepared at the evaporation rates of (a) $1.8 \mathrm{~g} \mathrm{~h}^{-1}$ and (b) $7.0 \mathrm{~g} \mathrm{~h}^{-1}$.

of an electric field for the composite films prepared at the evaporation rate of 1.8 and $7.0 \mathrm{~g} \mathrm{~h}^{-1}$ is also because of the difference in the anchoring effect for them. As mentioned above, with decreasing the size of LC domains, the fraction of LC molecules strongly anchored by polymer wall should be increased. As a result, the transmittance of the composite film prepared with the larger LC domain size was higher than that with smaller one, due to the larger fraction of oriented LC molecules along the direction of an applied electric field.

Figures 10(a) and (b) show the applied voltage dependence of the transmittance for the composite films prepared at the evaporation rates of $1.8 \mathrm{~g} \mathrm{~h}^{-1}$ and $7.0 \mathrm{~g} \mathrm{~h}^{-1}$, respectively. Two curves for reciprocating processes of the increasing and the decreasing an electric voltage are given in each plot. The reciprocating period was $10 \mathrm{~s}$. Obviously, an electric voltage to attain a higher transmittance, that is a driving voltage, for the composite film prepared at $1.8 \mathrm{~g} \mathrm{~h}^{-1}$ was lower than that at $7.0 \mathrm{~g} \mathrm{~h}^{-1}$. This also results from that the fraction of LC molecules strongly anchored by polymer walls in the composite film should be increased with decreasing the size of LC domains. Therefore, if a display material which can exhibit a lower voltage drive is required, the size of LC domains should be enlarged. The switching characteristics can be remarkably improved also by modifications of the kinds of materials, the composite fraction of the LC and polymer phases and the cell structure between two electrodes. The driving voltage less than $10 \mathrm{~V}$ has been achieved for the (Pdi-iPF/LC) composite system. That result will be 
reported elsewhere. ${ }^{23}$

\section{CONCLUSIONS}

The phase separation behavior of the composite film during the solvent evaporation could be classified into 3 stages based on the experimental results of the light scattering properties of the solution. In the second stage, the spinodal decomposition occurred between the polymer-rich phase and the LC-rich phase being still isotropic state. In the final stage, the anisotropic phase of LC generated in the solution. By regulating the evaporation rate in the second stage, the final domain size of LC in the composite film was controlled. With increasing the size of LC domains, though the rise response time and the driving voltage decreased, the decay response time increased. Those electro-optical results could be explained by the effective anchoring strength at the interface of polymer and LC depending on the effective area fraction of the interface in the composite film.

\section{REFERENCES}

1. T. Kajiyama, Y. Nagata, E. Maemura, and M. Takayanagi, Chem. Lett., 679 (1979).

2. T. Kajiyama, Y. Nagata, S. Washizu, and M. Takayanagi, $J$. Membrane Sci., 11, 39 (1982).

3. T. Kajiyama, S. Washizu, and M. Takayanagi, J. Appl. Polym. Sci., 29, 3955 (1984).

4. S. Washizu, I. Terada, T. Kajiyama, and M. Takayanagi, Polym.
J., 16, 307 (1984).

5. H. Kikuchi, M. Katayose, A. Takahara, S. Shinkai, and T. Kajiyama, Kobunshi Ronbunshu, 43, 669 (1986).

6. H. Kikuchi, A. Kumano, T. Kajiyama, M. Takayanagi, and S. Shinkai, J. Chem. Soc. Jpn., Chem. Ind. Chem., 423 (1987).

7. T. Kajiyama, H. Kikuchi, and S. Shinkai, J. Membrane Sci., 36 243 (1988).

8. H. G. Crighead, J. Cheng, and S. Hackwood, Appl. Phys. Lett., 40, 22 (1982).

9. J. L. Fergason, SID Int. Symp. Dig. Tech., 16, 68 (1985).

10. P. S. Drzaic, J. Appl. Phys., 60, 2142 (1986).

11. J. W. Doane, N. A. Vaz, B. G. Wu, and S. Zumer, Appl. Phys. Lett., 48, 27 (1986).

12. T. Kajiyama, A. Morimura, H, Kikuchi, and Y. Morimura, Chem. Lett., 1989, 813 (1989).

13. T. Kajiyama, H. Kikuchi, A. Miyamoto, S. Moritomi, and J. C. Hwang, Chem. Lett., 1989, 817 (1989).

14. H. Kikuchi, A. Miyamoto, S. Kobayashi, Y. Morimura, and T. Kajiyama, J. Chem. Soc. Jpn., Chem. Ind. Chem., 434 (1990).

15. A. Miyamoto, H. Kikuchi, Y. Morimura, and T. Kajiyama, New Polym. Mater., 2, 27 (1990).

16. A. Miyamoto, H. Kikuchi, S. Kobayashi, Y. Morimura, and T. Kajiyama, Macromolecules, 24, 3915 (1991).

17. K. Park, H. Kikuchi, and T. Kajiyama, Trans. Mater. Res. Soc. Jpn., 13, 18 (1993).

18. S. Tanioka, H. Kikuchi, and T. Kajiyama, Trans. Mater. Res. Soc. Jpn., 13, 28 (1993).

19. K. Park, H. Kikuchi, and T. Kajiyama, Polym. J., 26, 895 (1994).

20. J. W. Cahn, J. Chem. Phys., 422, 93 (1963).

21. J. W. Cahn and J. E. Hilliard, J. Chem. Phys., 28, 258 (1958).

22. P. G. de Gennes, "The Physics of Liquid Crystals," Oxford University, New York, N.Y., 1974, Chapter 3

23. H. Jeong, H. Kikuchi, and T. Kajiyama, Rept. Prog. Polym. Phys. Jpn., in press (1995). 